\title{
DNA microarray analysis of transcriptional responses of mouse spinal cords to physical exercise
}

\author{
Kazunori Hashimoto1, Akiko Honda², Yuichi Hayashi', Takashi Inuzuka1, Masahiko Satoh² \\ and Isao Hozumi ${ }^{1}$ \\ ${ }^{1}$ Department of Neurology and Geriatrics, Gifu University Graduate School of Medicine, 1-1 Yanagido, Gifu \\ 501-1194, Japan \\ ${ }^{2}$ Laboratory of Pharmaceutical Health Sciences, School of Pharmacy, Aichi Gakuin University, 1-100 Kusumotocho, \\ Chikusa-ku, Nagoya, Aichi 464-8650, Japan
}

(Received April 6, 2009; Accepted April 23, 2009)

\begin{abstract}
We present herein transcriptional changes in mouse spinal cords in response to physical exercise on a treadmill using a DNA microarray. By 30-min exercise, the expression of 3 genes was enhanced and expression of 29 genes reduced. By continuous 2-week exercise (30-min exercise per day), the expression of 1 gene was enhanced and expression of 13 genes reduced.
\end{abstract}

Key words: Exercise, Treadmill, Spinal cord, DNA microarray, Amyotrophic lateral sclerosis

\section{INTRODUCTION}

Recent studies suggest that physical exercise has a beneficial effect on the progression of amyotrophic lateral sclerosis (ALS) (McCrate and Kaspar, 2008). In addition, regular exercise can increase the lifespan of a transgenic mouse model of familial ALS (FALS) (G93A SOD1) and delay the onset (Kirkinezos et al., 2003). ALS is a progressive and fatal neurodegenerative disease, characterized by selective motor neuron degeneration. The majority of ALS cases are sporadic ALS (SALS), and approximately $10 \%$ of ALS cases are FALS, $10-20 \%$ of which are the result of mutations in the $\mathrm{Cu} / \mathrm{Zn}$ superoxide dismutase (SOD1) gene. Although the etiology of SALS remains unclarified, several possible mechanisms of motor neuron degeneration in SALS have been proposed, including oxidative stress, heavy metal toxicity, the conformational disorder of proteins and dysfunction of neurotrophic factors.

However, the mechanism by which exercise affects motor neurons is poorly understood. Herein, we examined how acute or continuous physical exercise affects gene expression in the spinal cords of normal mice on the 1 st day and the 14th day.

\section{MATERIALS AND METHODS}

\section{Animals and exercise}

C57BL/6J male mice were purchased from Japan SLC, Inc. (Shizuoka, Japan). Mice were housed in a temperature-controlled $\left(23 \pm 3^{\circ} \mathrm{C}\right)$ room with a 12-hr light/12$\mathrm{hr}$ dark cycle. Food and water were provided ad libitum throughout this study. The treadmill running exercise program was administered as described previously (Kirkinezos et al., 2003). Briefly, the male mice in the exercise groups ran on a treadmill machine (MK-680S, Muromachi, Tokyo, Japan) at $13 \mathrm{~m} / \mathrm{min}$ and $30 \mathrm{~min} /$ day. The exercise program was carried out from 9 to 10 o'clock. A control group remained sedentary. The exercise was started at 7 weeks of age. For transcriptional change analysis following $30 \mathrm{~min}$ of exercise stimulation, mice were anesthetized and sacrificed, and their spinal cords removed $12 \mathrm{hr}$ after exercise. On the other hand, for transcriptional change analysis following 2 weeks of 30 -min daily exercise, mice were prepared $24 \mathrm{hr}$ after the last exercise. The experimental designs and all procedures were in accordance with the National Institute of Health Guide for the Care and Use of Laboratory Animals and the guidelines on the handling of experimental animals issued by the Japanese Association for Laboratory Animal Science and the Animal Experimental Committee of Gifu University. This study was approved by the Animal Experimental Committee of Gifu University.

Correspondence: Isao Hozumi (E-mail: metallo@gifu-u.ac.jp) 
K. Hashimoto et al.

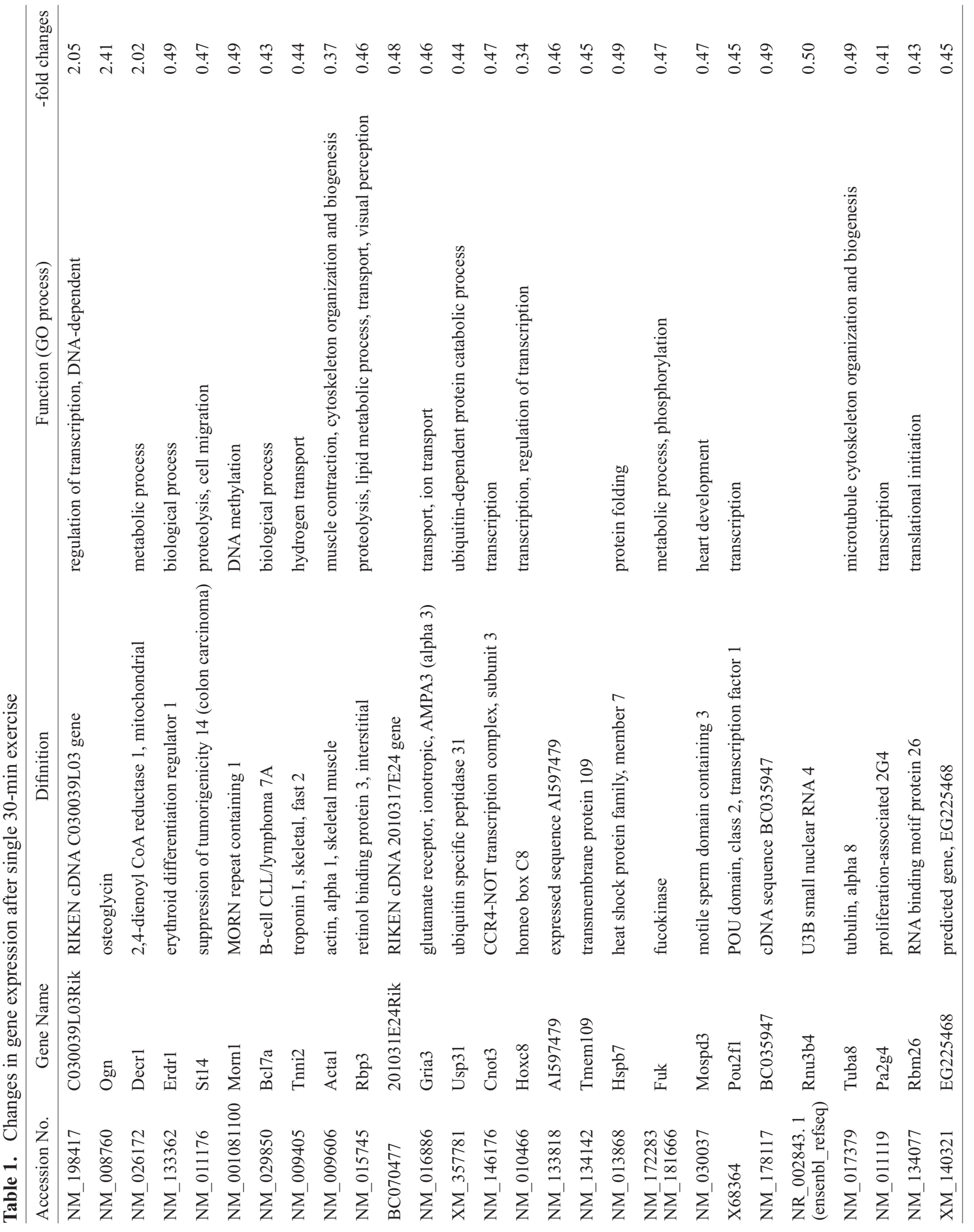

Vol. 34 No. 4 
Responses of spinal cord to exercise
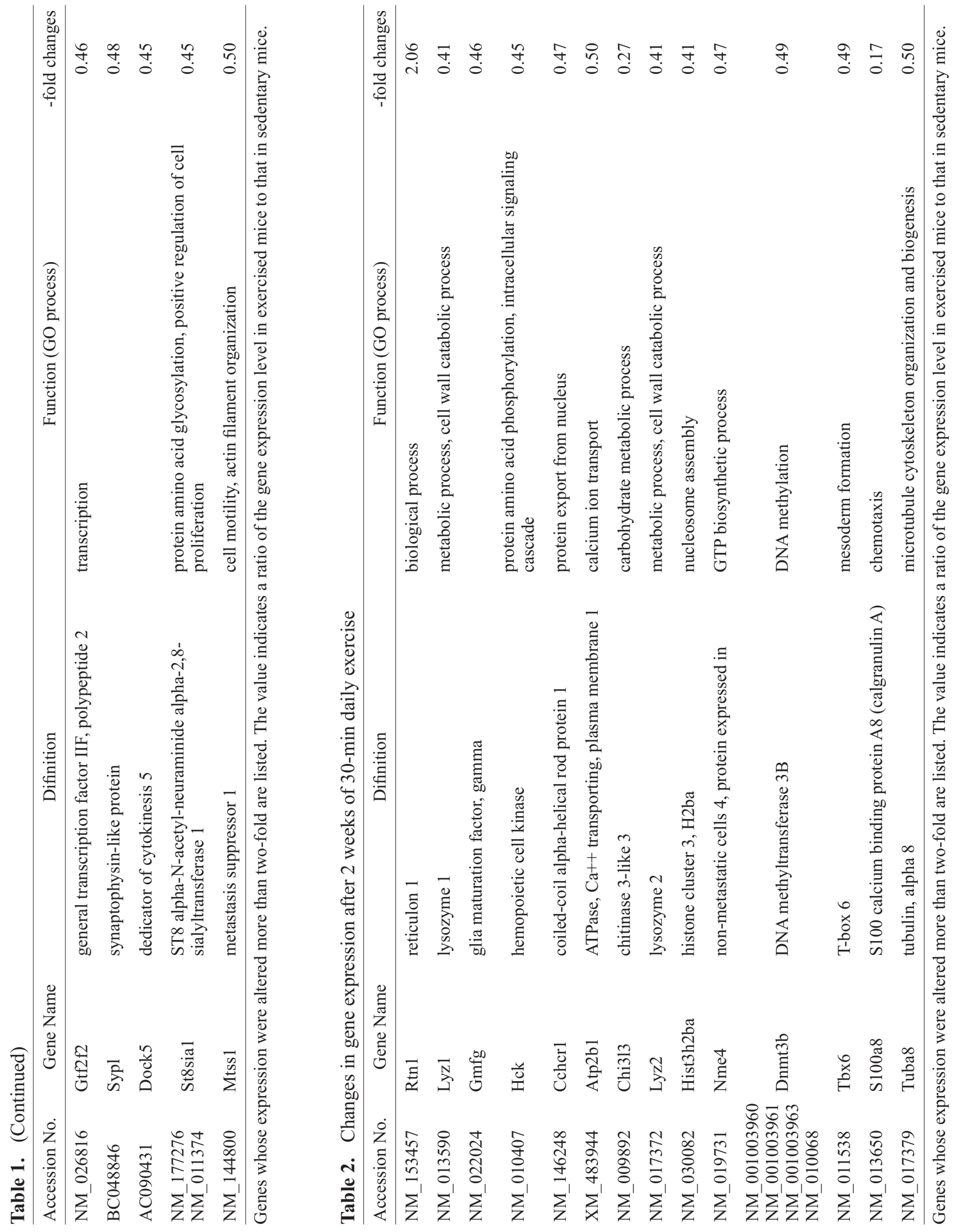

Vol. 34 No. 4 


\section{Preparation of RNAs for DNA microarray analysis}

Total RNAs were isolated from each of 4 or 5 mice spinal cords per exercised or sedentary group using the RNeasy Lipid Tissue Mini Kit (Qiagen GmbH, Hilden, Germany). Pooled total RNA ( $5 \mu \mathrm{g}$ each, 4 or 5 mice per group) was applied to OpArray ${ }^{\mathrm{TM}}$ Mouse V4.0 (Operon Technologies, Alameda, CA, USA), in which 35,852 genes are registered. We used Low RNA Fluorescent Linear Amp Kit PLUS (Agilent Technologies Inc., Santa Clara, CA, USA) to synthesize double-stranded (ds) cDNA from the total RNA and then synthesize cRNA from the ds cDNA as a template. Total RNA was reversetranscribed with a T7 Promoter primer. After secondstrand synthesis, we used the ds cDNA as a template for Cyanine (Cy) 3/Cy5-labeled cRNA synthesis. The OpArray slide was hybridized with $20-25 \mu \mathrm{g}$ of each labeled cRNA and washed with the OpArray Hybridization Buffer Kit (Operon). A fluorescent image of the OpArray slide was then recorded with CRBIO (Hitachi Software Engineering, Tokyo, Japan). The digitized image data were processed with DNASIS Array software (Hitachi Software Engineering). The ratios of intensity of Cy5 to that of $\mathrm{Cy} 3$ were calculated. Information on each gene on the slide was obtained from the National Center for Biotechnology Information (NCBI) database.

\section{RESULTS AND DISCUSSION}

This study demonstrated that single 30-min or 2-week exercise resulted in specific gene expression changes in mouse spinal cords using microarrays. By 30-min exercise, the expression of 3 genes was enhanced, and the expression of 29 genes reduced (Table. 1). By continuous 2-week exercise, the expression of 1 gene was enhanced, and the expression of 13 genes was reduced (Table. 2). Gria3 is glutamate receptor called GluR3, whose antisense peptide nucleic acid targeting GluR3 delayed disease onset and progression in the SOD1 G93A mouse model of familial ALS (Rembach et al., 2004). The elimination of St8sial coding GD3 synthase improved memory and reduced amyloid-beta plaque load in Alzheimer disease model mice (Bernardo et al., 2008). Reticulon family members including Rtn 1 coding reticulon 1 modulated BACE1 activity and amyloid-beta peptide generation (He et al., 2004). This study will provide a valuable clue on the molecular basis of exercise-induced neuroprotective effects in spinal cords.

\section{ACKNOWLEDGMENTS}

This work was supported by grants from the Ministry of Education, Culture, Sports, Science and Technology of Japan (Basic Research (B) 19390151 (to I.H.), Young Scientists (B) 19790603 (to Y.H.)).

\section{REFERENCES}

Bernardo, A., Harrison, F.E., McCord, M., Zhao, J., Bruchey, A., Davies, S.S., Jackson, Roberts, L.2nd., Mathews, P.M., Matsuoka, Y., Ariga, T., Yu, R.K., Thompson, R. and McDonald, M.P. (2008): Elimination of GD3 synthase improves memory and reduces amyloid-beta plaque load in transgenic mice. Neurobiol. Aging. [Epub ahead of print]

He, W., Lu, Y., Qahwash, I., Hu, X.Y., Chang, A. and Yan, R. (2004): Reticulon family members modulate BACE1 activity and amyloid-beta peptide generation. Nat Med., 10, 959-965.

Kirkinezos, I.G., Hernandez, D., Bradley, W.G. and Moraes, C.T. (2003): Regular exercise is beneficial to a mouse model of amyotrophic lateral sclerosis. Ann Neurol., 53, 804-807.

McCrate, M.E. and Kaspar, B.K. (2008): Physical activity and neuroprotection in amyotrophic lateral sclerosis. Neuromol. Med., 10, 108-117.

Rembach, A., Turner, B.J., Bruce, S., Cheah, I.K., Scott, R.L., Lopes, E.C., Zagami, C.J., Beart, P.M., Cheung, N.S., Langford, S.J. and Cheema, S.S. (2004): Antisense peptide nucleic acid targeting GluR3 delays disease onset and progression in the SOD1 G93A mouse model of familial ALS. J Neurosci Res., 77, 573582. 